\title{
Pulmonary hepatic flow distribution in total cavopulmonary connections: Extracardiac versus intracardiac
}

\author{
Lakshmi P. Dasi, PhD, ${ }^{a}$ Kevin Whitehead, PhD, ${ }^{\mathrm{b}}$ Kerem Pekkan, ${ }^{\mathrm{c}}$ Diane de Zelicourt, MS, ${ }^{\mathrm{d}}$ \\ Kartik Sundareswaran, $\mathrm{PhD},{ }^{\mathrm{d}}$ Kirk Kanter, MD, ${ }^{\mathrm{e}}$ Mark A. Fogel, MD, ${ }^{\mathrm{b}}$ and Ajit P. Yoganathan, $\mathrm{PhD}^{\mathrm{d}}$
}

Objective: Pulmonary arteriovenous malformations can occur after the Fontan procedure and are believed to be associated with disproportionate pulmonary distribution of hepatic venous effluent. We studied the effect of total cavopulmonary connection geometry and the effect of increased cardiac output on distribution of inferior vena caval return to the lungs.

\begin{abstract}
Methods: Ten patients undergoing the Fontan procedure, 5 with extracardiac and 5 with intracardiac configurations of the total cavopulmonary connection, previously analyzed for power loss were processed for calculating the distribution of inferior vena caval return to the lungs (second-order accuracy). One idealized total cavopulmonary connection was similarly analyzed under parametric variation of inferior vena caval offset and cardiac output flow split.

Results: Streaming of the inferior vena caval return in the idealized total cavopulmonary connection model was dependent on both inferior vena caval offset magnitude and cardiac output flow-split ratio. For patient-specific total cavopulmonary connections, preferential streaming of the inferior vena caval return was directly proportional to the cardiac output flow-split ratio in the intracardiac total cavopulmonary connections $(P<.0001)$. Preferential streaming in extracardiac total cavopulmonary connections correlated to the inferior vena caval offset $(P<.05)$ and did not correlate to cardiac output flow split. Enhanced mixing in intracardiac total cavopulmonary connections is speculated to explain the contrasting results. Exercising tends to reduce streaming toward the left pulmonary artery in intracardiac total cavopulmonary connections, whereas for extracardiac total cavopulmonary connections, exercising tends to equalize the streaming.
\end{abstract}

Conclusions: Extracardiac and intracardiac total cavopulmonary connections have inherently different streaming characteristics because of contrasting mixing characteristics caused by their geometric differences. Pulmonary artery diameters and inferior vena caval offsets might together determine hepatic flow streaming. (J Thorac Cardiovasc Surg 2011;141:207-14)

Pulmonary arteriovenous malformations (PAVMs), also referred to as pulmonary arteriovenous fistulas, can occur after the Fontan procedure, resulting in decreased systemic oxygenation. PAVMs are intrapulmonary arterial to venous shunts in which the systemic venous blood reaches the pulmonary venous system through abnormal vascular connections proximal to the gas exchange units. The primary consequence

From the Department of Mechanical Engineering \& School of Biomedical Engineering, ${ }^{a}$ Colorado State University, Fort Collins, Colo; the Division of Cardiology, ${ }^{\text {b }}$ Children's Hospital of Philadelphia, Philadelphia, Pa; Department of Biomedical Engineering, ${ }^{c}$ Carnegie Mellon University, Pittsburgh, Pa; the Wallace H. Coulter School of Biomedical Engineering, ${ }^{\mathrm{d}}$ Georgia Institute of Technology, Atlanta, $\mathrm{Ga}$; and Emory University, ${ }^{\mathrm{e}}$ Atlanta, Ga.

Supported by Bioengineering Research Partnership grant from National Institutes of Health (HL67622). Dr Whitehead was supported in part by National Institutes of Health training grant T32 HL007915-08.

Disclosures: Authors have nothing to disclose with regard to commercial support.

Received for publication Dec 22, 2009; revisions received May 20, 2010; accepted for publication June 6, 2010; available ahead of print July 12, 2010.

Address for reprints: Ajit P. Yoganathan, PhD, Wallace H. Coulter School of Biomedical Engineering, Georgia Institute of Technology and Emory University, Room 2119 U. A. Whitaker Building, 313 Ferst Dr, Atlanta, GA 30332-0535 (E-mail: ajit.yoganathan@bme.gatech.edu).

0022-5223/\$36.00

Copyright (c) 2011 by The American Association for Thoracic Surgery

doi:10.1016/j.jtcvs.2010.06.009 of PAVMs is decreased systemic oxygen saturation. The prognosis for patients with PAVMs undergoing the Fontan procedure is often poor without reoperation to reconstruct the Fontan baffle. PAVMs have been postulated in the past to be secondary to disproportionate distribution of hepatic factors between the 2 lungs. ${ }^{1-5}$ More recent studies confirmed that geometric configuration of the total cavopulmonary connection (TCPC) to preferentially stream hepatic venous flow to a single lung leads to PAVMs. ${ }^{6}$ Thus it is important to determine TCPC geometric characteristics necessary to avoid preferential hepatic venous flow streaming. A recent study underscores the importance of geometric characteristics in a particularly complex case of PAVMs. ${ }^{7}$

The TCPC is a double-inlet, double-outlet connection that routes the venous return from the superior vena cava (SVC) and inferior vena cava (IVC) to the left pulmonary artery (LPA) and the right pulmonary artery (RPA). Although most previous hemodynamic studies of the TCPC have focused on the power loss characteristics, ${ }^{8-12}$ flow streaming might be an equally important characteristic, specifically during the early growing period of the lung after conversion from Glenn stage to a Fontan connection. Thus one can postulate that an ideal TCPC needs to impose the least hemodynamic 


$$
\begin{aligned}
& \text { Abbreviations and Acronyms } \\
& \text { CO = cardiac output } \\
& \text { IVC = inferior vena cava } \\
& \text { LPA = left pulmonary artery } \\
& \text { PAVM = pulmonary arteriovenous malformation } \\
& \text { RPA = right pulmonary artery } \\
& \text { SVC = superior vena cava }
\end{aligned}
$$

resistance (for minimal power losses), as well as facilitate balanced left and right streaming of inferior venous return for sufficient hepatic factor transport to both lungs.

In this study we quantify the flow branching of inferior blood returning through the Fontan baffle to the pulmonary arteries in the 2 major types of TCPCs, namely extracardiac and intracardiac TCPCs. Of particular interest is the following question: What are the geometric parameters of TCPCs (eg, pulmonary artery diameters and vessel offset) that can be controlled during the construction of a Fontan TCPC to avoid preferential streaming of hepatic venous flow? The answer to this question has strong clinical implications from the standpoint of choosing a particular TCPC type along with an understanding of the desired geometric characteristics for reducing the risk of PAVM formation. Also, it provides another perspective given the substantial literature on power loss characteristics of the TCPC and its correlation with the geometry of the connection.

\section{MATERIALS AND METHODS}

The blood-flow fields through 10 patient-specific TCPC geometries, as well as an idealized TCPC geometry, were computed by using computational fluid dynamics tools and then analyzed to calculate the distribution of the IVC flow between the left and right lungs. The results were then examined to correlate TCPC geometry type (extracardiac or intracardiac) and influence of exercise on the distribution/streaming characteristics.

\section{Patients' Data}

Ten patients, 5 each with extracardiac and intracardiac TCPCs, were selected from the Georgia Tech MRI database of patients undergoing the Fontan procedure (http://fontan.bme.gatech.edu). The database is part of a National Institutes of Health-funded ongoing study for understanding Fontan hemodynamics. The demographics of the 10 patients are shown in Table 1. All patients were imaged at Children's Hospital of Philadelphia. Informed consent was obtained from all patients, and all study protocols complied with the Institutional Review Boards of the Children's Hospital of Philadelphia and the Georgia Institute of Technology. The inclusion criteria for this study were as follows: (1) availability of axial magnetic resonance images to reconstruct the TCPC and aortic arch, (2) availability of clinical information necessary to categorize each study group, and (3) availability of computational results under resting and simulated exercise conditions at equal pulmonary lung resistance conditions. For all these criteria, 10 was the maximum of more than 200 patients in the database. For all 10 patients, the anatomy of the TCPC was reconstructed by using previously described segmentation and reconstruction techniques. ${ }^{13,14}$ Geometric offsets of the IVC relative to the SVC were calculated for each of the patientspecific TCPCs from previously described methods. ${ }^{15}$ There was no signif- icant difference in cardiac output $(\mathrm{CO})$ between the 2 groups of patients, and the mean age of both groups was above the threshold ${ }^{16}$ for any variation in SVC/IVC flow ratio.

\section{Computational Model}

Computational fluid dynamics simulations were conducted with FIDAP (ANSYS, Inc, Canonsburg, Pa) for each TCPC. Some of the data, along with the detailed description of the simulation parameters, have been published previously in the context of energy loss characteristics of the TCPC. ${ }^{17}$ The flow conditions for each of the models correspond to the resting conditions and 2 simulated levels of exercise, namely the $2 \mathrm{x}$ and $3 \mathrm{x}$ resting flow conditions. ${ }^{17}$ The $\mathrm{CO}$ flow split is defined as the fraction of the net inflow streaming to the LPA. In these simulations the CO flow split for each patient corresponds to imposed equal pulmonary lung resistance condition. Imposing this condition ensures that the difference in the split of $\mathrm{CO}$ between the 2 lungs is purely governed by the resistance of the TCPC and not from differences in left and right pulmonary vascular bed resistances. This is an important condition to ensure that hepatic venous flow streaming characteristics are not biased by patient-to-patient variation in the difference between left and right lung resistance. Results on the idealized TCPC model are also presented for varying flow split and offset of the IVC from the SVC to help develop insight into the flow-split physics.

\section{Flow-Split Computation}

Figure 1 shows a schematic that explains the methodology for flow-split computation on an idealized 1-dimensional offset TCPC. Ten thousand particles were uniformly seeded in a cross-section of the IVC. These particles have zero mass, and their trajectories were computed by integrating the following equation of motion for each particle, using a second-order RungeKutta numeric method:

$$
\begin{aligned}
& \dot{x}=u(x, y, z) \\
& \dot{y}=v(x, y, z) \\
& \dot{z}=w(x, y, z),
\end{aligned}
$$

where $u, v$, and $w$ are the 3-dimensional velocity components obtained from the computational model for each particle. Note that only the steady-state computational fluid dynamics solutions were considered for this analysis. Therefore the velocity is not a function of time. $\dot{x}, \dot{y}$, and $\dot{z}$ are the time derivatives of the actual 3-dimensional particle position at time $t$ given by $\mathrm{x}(t)$, $\mathrm{y}(t)$, and $\mathrm{z}(t)$.

Figure 2, $A$, shows a plot of all the trajectories calculated for the seeded particles in Figure 1. Each trajectory is sorted based on whether the particle is bound to the left lung or the right lung, respectively. Based on the fate of the particle trajectory, the cross-section shown in Figure 1 is then partitioned into left-bound or right-bound areas. Examples of partitioning are shown in Figure 2, $B$. The corresponding flow distribution is then calculated by integrating the velocity over the partitioned domains. Equations are shown at the 2 outlets of the LPA and RPA in Figure 1. The end result is the estimation of the exact split of the blood returning from the IVC to the 2 lungs. We note here that we assume the hepatic factor or factors to be uniformly mixed with the IVC blood. This calculation is denoted as hepatic venous flow split and is different from CO flow split in the sense that it is the split of IVC flow alone to the LPA.

\section{Normalization}

The CO flow split to the LPA is normalized by $\mathrm{CO}$, and the estimated hepatic venous flow split to the LPA is normalized to the total IVC flow. Therefore a $50 \%$ value indicates equal streaming.

\section{Statistical Analysis}

Because the data were nonnormally distributed and corresponded to a 2-sample population (extracardiac vs intracardiac), the nonparametric Mann-Whitney test was used to examine statistical significance among 
TABLE 1. Summary of differences in demographics and cardiac output for the EC and IC geometries

\begin{tabular}{lcccc}
\hline & All & EC & IC & P value \\
\hline Age $(\mathrm{y})$ & $10.2 \pm 4.7$ & $6.6 \pm 2.2$ & $13.2 \pm 3.7$ & $<.01$ \\
BSA $\left(\mathrm{m}^{2}\right)$ & $1.14 \pm 0.4$ & $0.78 \pm 0.2$ & $1.4 \pm 0.28$ & $<.01$ \\
CO $(\mathrm{L} / \mathrm{min})$ & $2.9 \pm 1.0$ & $2.6 \pm 1.1$ & $3.4 \pm 0.7$ & .17 \\
CI $\left(\mathrm{L} \cdot \mathrm{min}^{-1} \cdot \mathrm{m}^{-2}\right)$ & $2.7 \pm 0.4$ & $2.9 \pm 0.4$ & $2.5 \pm 0.3$ & .11 \\
SRV & 4 & 1 & 3 & \\
SLV & 6 & 4 & 2 & \\
\hline
\end{tabular}

Modified from Whitehead and colleagues. ${ }^{17} E C$, Extracardiac; $I C$, intracardiac; $B S A$, body surface area; $C O$, cardiac output; $C I$, cardiac index; $S R V$, single right ventricle; $S L V$, single left ventricle.

the various parameters evaluated. Standard regression analysis is used to assess functional dependence where a $P$ value of less than .05 indicates a statistically significant functional dependence.

\section{RESULTS}

The results include calculation of the hepatic venous flow split for the idealized TCPC, as well as the 10 patientspecific TCPCs. For the idealized TCPC, the hepatic venous flow split is calculated for varying offset between the IVC and SVC (from 0.4D to D, where the IVC is offset by 1 caval diameter [D] toward the RPA) and varying CO split (ie, percentage of CO directed toward the LPA). For the patientspecific TCPCs, hepatic venous flow split is calculated for the resting and 2 simulated exercise conditions $(2 \mathrm{x}$ and $3 \mathrm{x})$ all at equal pulmonary resistance conditions.

\section{Idealized TCPC}

Figure 2, $A$, depicts the preferential streaming of inferior venous return to the right lung in a simplified 1-dimensional

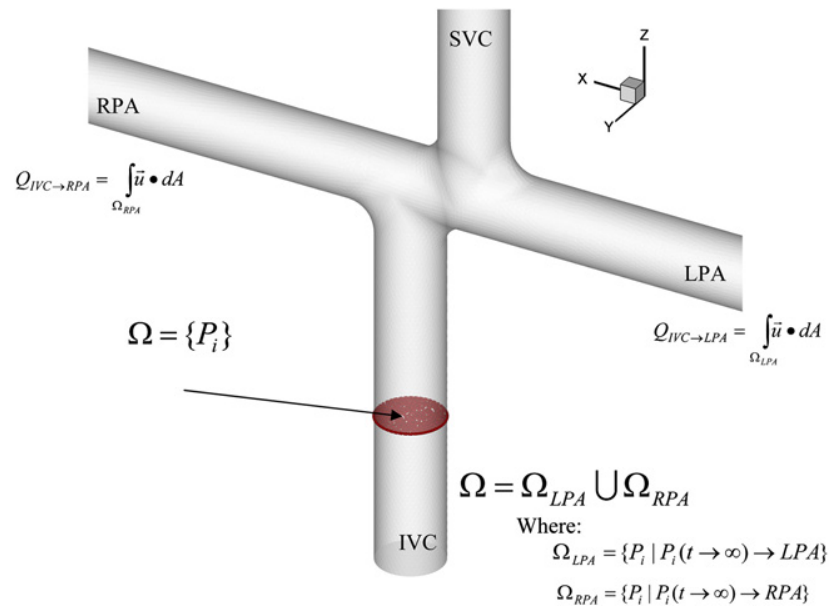

FIGURE 1. Mathematic definition of inferior venous split to the 2 pulmonary arteries depicted on a simplified 1-dimensional offset idealization of the total cavopulmonary connection. Ten thousand mass-less particles denoted as set $\left\{P_{i}\right\}$ are seeded in the inferior vena cava (IVC) and tracked by using the velocity field output of the computational model. The seeding cross-section is then partitioned into $\Omega$ left pulmonary artery ( $\Omega L P A)$ and $\Omega$ right pulmonary artery $(\Omega R P A)$ based on the fate of the particle after it transits through the connection. $S V C$, Superior vena cava.

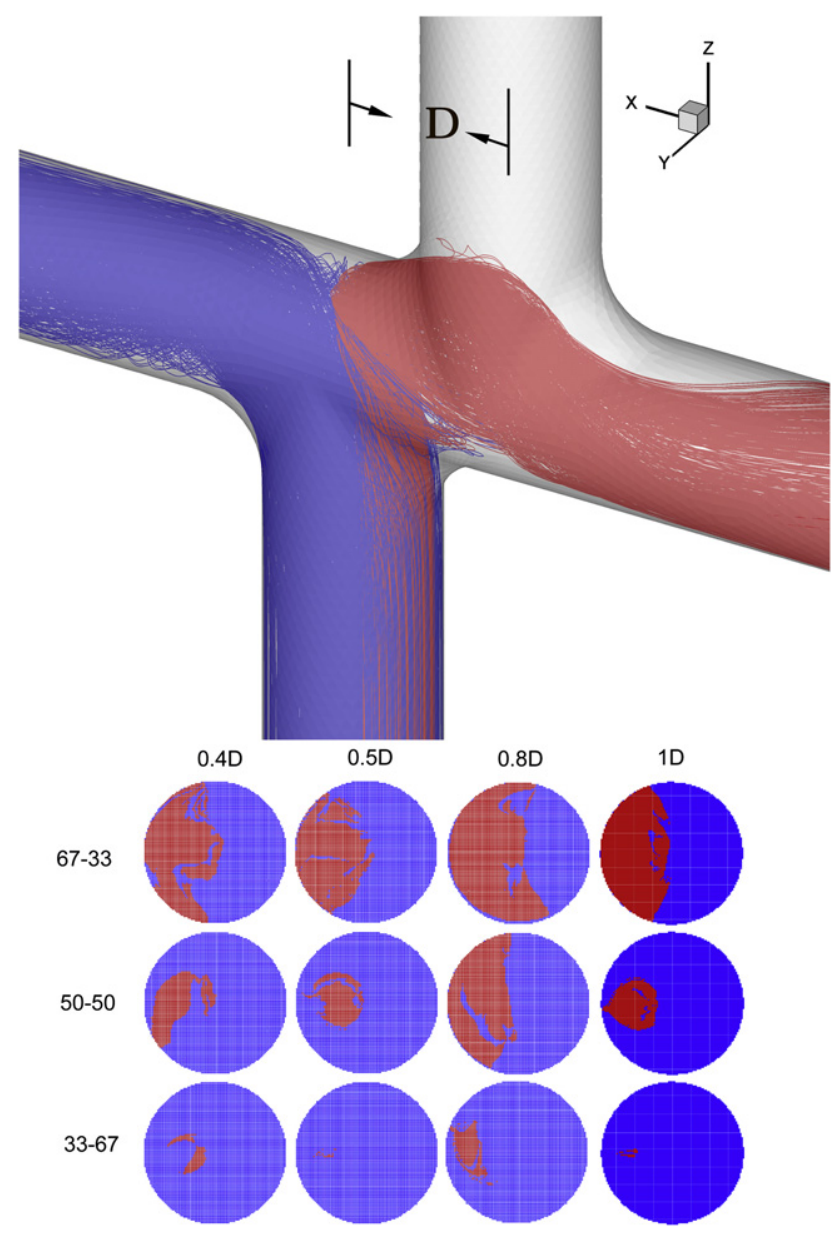

FIGURE 2. A, Visualization of streamlines color coded (red, flow bound to the left lung; blue, flow bound to the right lung) on the 1-dimensional offset model. B, Partitioning the seeding cross-section of the inferior vena cava (shown in Figure 1) to left- and right bound-flow distribution for varying cardiac output flow split and the variation of offset.

offset model of the TCPC. The topologic changes in the preferential streaming are further depicted in Figure 2, $B$, showing the cross-section on the IVC in which passive tracers were seeded and color coded to right PA-bound and left PA-bound streams. These results show that the structure of the partitions changes significantly with both the examined parameters, namely offset and $\mathrm{CO}$ flow split.

\section{Patient-Specific TCPCs}

Shown in Figures 3 and 4 are the streaming details and normalized flow partitioning data obtained for the 5 intracardiac and 5 extracardiac TCPCs, respectively. The patients are labeled by their database code (CHOPxxx). These results show that the flow-partitioning structure from patient to patient is highly variable. Furthermore, the geometric structure of the partitioned cross-sections qualitatively remains similar between resting and simulated levels of exercise, with only a gradual variation in hepatic venous 


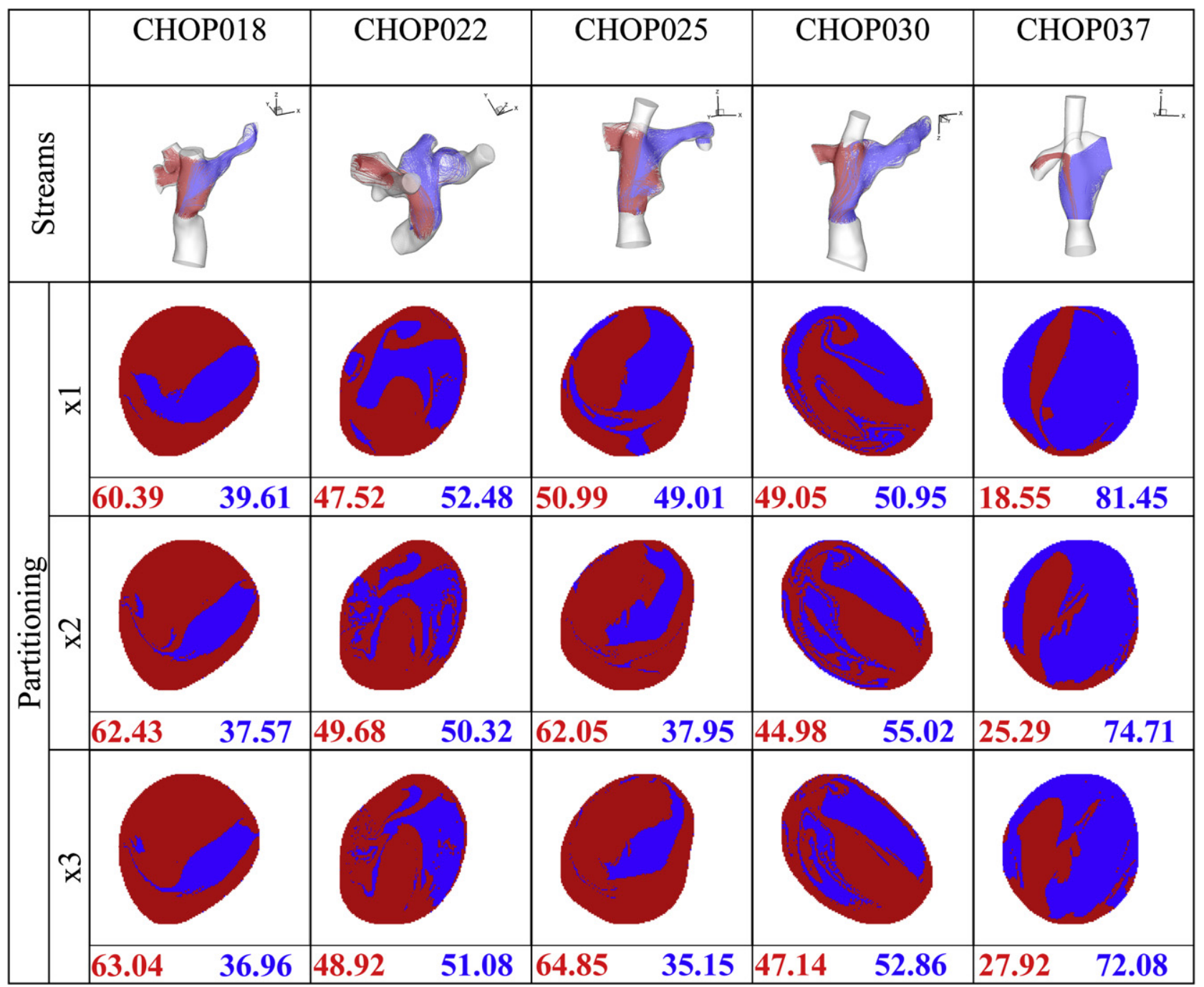

FIGURE 3. The 5 patient-specific intracardiac total cavopulmonary connections depicting flow streaming at resting conditions (top row). Also shown for each patient is the color-coded partitioning at the particle seeding cross-section of the inferior vena cava as a function of simulated exercise level. Blue, Leftbound flow; red, right-bound flow. CHOP, Children's Hospital of Philadelphia.

flow-split magnitude. Also subjectively, the geometric structure of the partition for the intracardiac TCPCs has a finer scale (more convoluted, folded, and often fractal like) than that for extracardiac TCPCs.

Figure 2 qualitatively showed a significant dependence of hepatic venous flow partitioning with $\mathrm{CO}$ flow split for the idealized TCPC. Figure 5 explores this dependence for the studied patient-specific cases. Note that because of imposed equal lung resistance conditions for the patient-specific TCPCs, any variations observed in the $\mathrm{CO}$ flow split are purely due to the directional resistance and geometric properties of the patient-specific TCPCs. Data points for both resting and simulated exercise conditions have been plotted in Figure 5. From this figure, it is clear that intracardiac TCPCs have a near-linear correlation, with a $P$ value of less than .0001 and an $R^{2}$ value of 0.93 between hepatic venous flow split and
CO flow split. The slope and intercept of the linear relationship are 0.84 ( \pm 0.07$)$ and 15.02 ( \pm 3.30$)$, respectively; quantities in parentheses correspond to the standard error. No significant correlation was found for the extracardiac TCPCs.

Figure 6 investigates the effect of IVC offset in extracardiac TCPCs. A significant correlation existed with a decrease in LPA streaming and increasing IVC offset toward the RPA $\left(P<.01\right.$ and $\left.R^{2}>0.91\right)$. The slope of the correlation did not significantly change with exercise.

Figure 7 shows the effect of exercise on the streaming characteristics. No statistically significant trends could be detected. However, it appears that an increase in $\mathrm{CO}$ mildly reduced $\%$ streaming to LPA in intracardiac TCPCs, whereas for extracardiac TCPCs, exercising tended to mitigate any preferential streaming with the \% LPA approaching the 50/ 50 value asymptotically. 


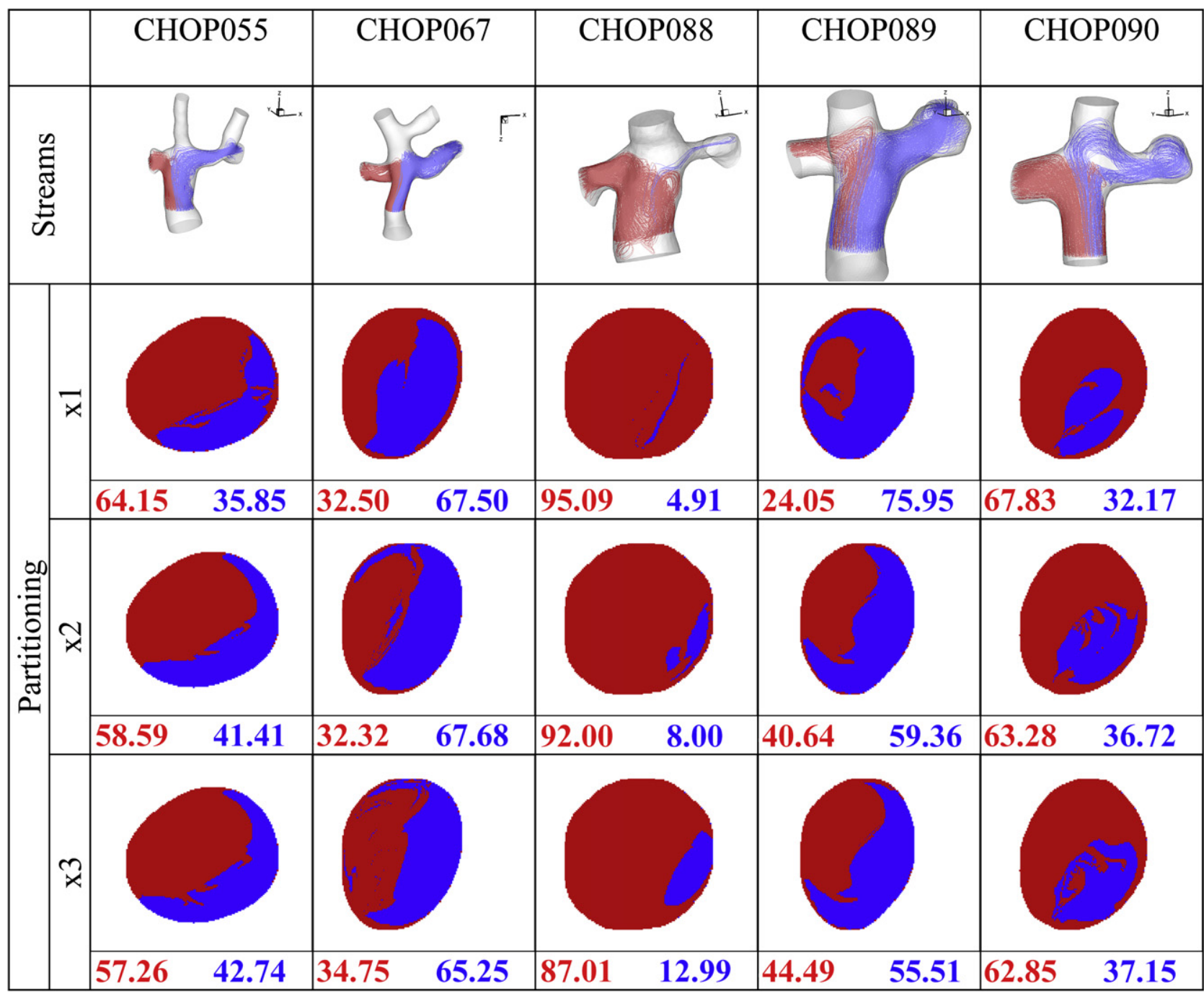

FIGURE 4. The 5 patient-specific extracardiac TCPCs depicting flow streaming at resting conditions (top row). Also shown for each patient is the partitioning at the particle seeding cross-section of the inferior vena cava as a function of simulated exercise level. Blue, Left-bound flow; red, right-bound flow. CHOP, Children's Hospital of Philadelphia.

\section{DISCUSSION}

This study, to the best of our knowledge, is the first that analyzes hepatic venous flow streaming to the lungs with respect to geometric characteristics of the TCPC and examines any influence exercise might have on hepatic venous streaming. The problem is highly relevant because surgeons have the opportunity during the construction of the TCPC to ensure adequate distribution of hepatic venous flow to both the lungs, as well as to minimize any hemodynamic energy losses. Failure to construct an optimal TCPC might result in the need for Fontan revision, in which the palliative strategy for correction of PAVMs typically involves attempts to redirect the hepatic effluent to the affected lung. Several approaches have been proposed to accomplish this objective, including a transcatheter reconnection, ${ }^{18}$ formation of arteriovenous fistulae, ${ }^{19}$ rerouting of the hepatic venous flow to the hemiazygos vein, ${ }^{4,20}$ redirection of the hepatic venous flow to the innominate vein, ${ }^{21}$ and the use of a bifurcated extracardiac conduit, ${ }^{22}$ to name a few. Clearly, the wide variety of patients' anatomies makes it difficult to design a general procedure that will suit all patients. Additionally, the complexity of patients' anatomies poses significant challenges to identify the surgical option that will best distribute hepatic flow for a given patient. Below we discuss the above-presented results and focus on the following $2 \mathrm{ob}$ jectives of this study: (1) the influence of TCPC geometry and $\mathrm{CO}$ flow split and (2) the influence of exercising.

\section{Influence of TCPC Geometry and CO Flow Split on Hepatic Venous Flow Streaming}

It is clear from the results that both geometry and $\mathrm{CO}$ flow split play a crucial role in hepatic venous flow streaming. 


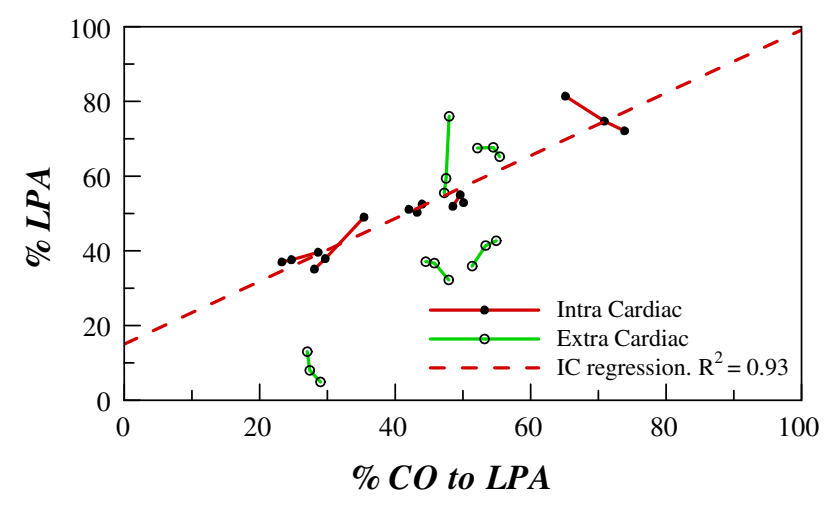

FIGURE 5. Inferior vena caval to left pulmonary artery $(L P A)$ streaming as a function of fraction of cardiac output $(\mathrm{CO})$ to the LPA for all 10 patients at resting and simulated conditions (connected symbols). Intracardiac (IC) patients showed a significant linear correlation $\left(P<.0001, R^{2}=0.93\right.$, dashed line), whereas extracardiac patients did not show significance $(P>.05)$.

Intuitively, this is obvious. Any change in geometric conditions or flow conditions is bound to change the flow field and hence affect how streams of hepatic factors interact with the SVC flow. These interactions include mixing, as well as splitting into left-bound and right-bound flows.

For the idealized TCPC, we studied the geometric change corresponding to the IVC offset and the CO flow split as independent parameters (Figure 2). As expected, both these parameters changed the partitioning (Figure 2, $B$ ). These results can be explained as follows. The tendency of the IVC flow to stream preferentially to the RPA is due to the "momentum barrier" created by the opposing SVC flow. In other words, the IVC flow needs to cross the SVC stream to reach the LPA side. Therefore the more the IVC is offset, the more difficult it is for hepatic venous flow to overcome the barrier and stream to the LPA. This effect is amplified when the CO preferentially splits to the RPA. Therefore for the idealized TCPC, both the independent parameters had a significant influence on the hepatic venous flow streaming. The results for the patient-specific TCPCs, as described below, are more interesting and are quantitatively analyzed.

Figure 5 clearly shows a drastic difference between intracardiac and extracardiac streaming characteristics. Specifically, preferential streaming in intracardiac TCPCs is directly proportional to the $\mathrm{CO}$ flow split, as depicted by the linear relationship. In contrast, extracardiac TCPCs did not significantly depend on the CO flow split. This is an interesting observation, and the fluid dynamics explanation is that it might be attributed to mixing between the SVC and IVC streams, which is higher in intracardiac TCPCs and lower in extracardiac TCPCs. The fact that there is more mixing in intracardiac TCPCs compared with that seen in extracardiac TCPCs is evidenced by the presence of finerscale structures in the partitions shown in Figure 3 compared with those shown in Figure 4. Notice the presence of struc-

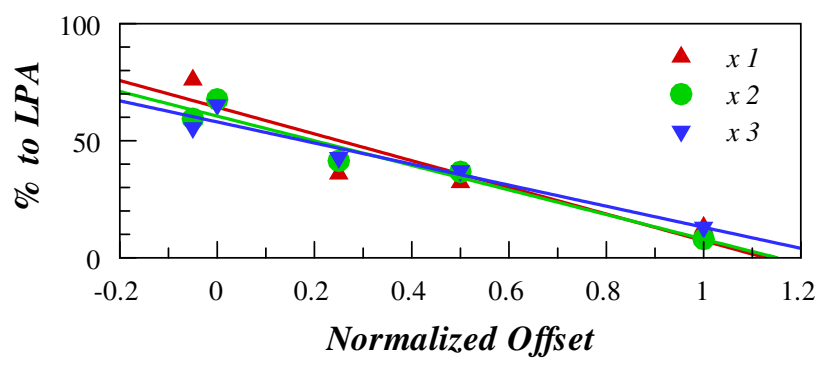

FIGURE 6. Left pulmonary artery ( $L P A)$ streaming as a function of inferior vena caval offset toward the right pulmonary artery in extracardiac total cavopulmonary connections. Offset is normalized to inferior vena caval diameter. Linear regression results in an $R^{2}$ value of greater than 0.91 and a $P$ value of less than .01 for each of the fitted lines. The slight decrease in slope with increasing cardiac output was not statistically significant.

tures resembling a stirring effect (analogous to the mixing of creamer when poured in black coffee). Qualitatively, these structures resembling mixing are much reduced in extracardiac TCPCs in Figure 4. Theoretically, if the SVC and IVC streams perfectly mix, then the hepatic venous flow split would be exactly equal to the CO flow split. Based on this observation, it might be presumed that any streaming tendencies in intracardiac TCPCs are governed by the CO flow split, which in turn is known to be related to the directional resistance of the TCPC.

From our previous studies, ${ }^{23,24}$ it is known that both $\mathrm{CO}$ and the $\mathrm{CO}$ split are primarily correlated to the pulmonary artery diameters. Therefore a corollary is that for the intracardiac TCPCs, streaming is proportional to the pulmonary artery diameter. In other words, more hepatic factors will go to the bigger pulmonary artery.

Given that there is no significant mixing in extracardiac TCPCs, the streaming is more sensitive to the relative orientation between the IVC and the SVC. As shown in Figure 6, the hepatic venous flow streaming characteristics of extracardiac TCPCs primarily depend on the IVC offset. Notice that as the IVC offset increases, hepatic venous flow streaming toward the LPA decreases. This is also consistent with the results shown in Figure 2 for an idealized extracardiac TCPC model. The fluid dynamics explanation is the same as that given for the idealized TCPC. The SVC flow creates a momentum barrier for the flow from the IVC to reach the LPA side. As confirmed from Figure 6, increase in offset reduces the net hepatic factors streamed toward the left lung. Figure 6 also shows that exercising does not appear to alleviate preferential streaming in extracardiac TCPCs. This is supported by the observation that the slope of the linear regression lines between the resting and simulated exercise conditions were not statistically different. These results combined with previous studies on power loss characteristics show that for the extracardiac TCPCs, offsetting the IVC is a tradeoff problem between power loss and hepatic venous streaming. 


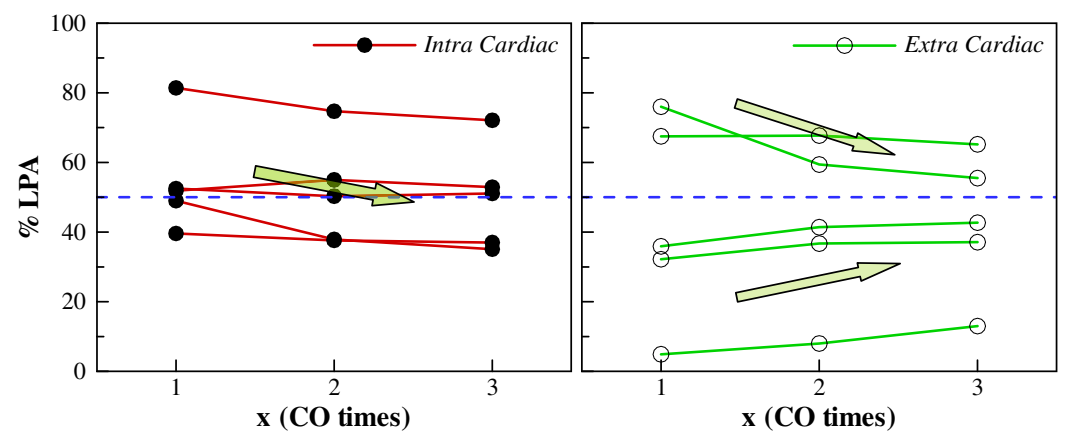

FIGURE 7. Effect of exercise on left pulmonary artery $(L P A)$ streaming characteristics of intracardiac and extracardiac total cavopulmonary connections shown separately. For intracardiac total cavopulmonary connections, the overall streaming decreases marginally with exercise. For extracardiac total cavopulmonary connections, streaming appears to tend to a value of $50 \%$ streaming with exercise. $\mathrm{CO}$, Cardiac output.

\section{Influence of Exercise on Hepatic Venous Flow Streaming}

Exercising is an important factor when it comes to the power loss characteristics of the TCPC. ${ }^{25}$ However, the present study shows that exercise has only a marginal effect on hepatic venous streaming characteristics with no statistical significance, as seen in Figure 7. At best, it appears that exercising has the following slight influence on streaming. For intracardiac TCPCs, exercise always reduces streaming to the LPA. This might be related to the fact that the LPA is often a little smaller than the RPA, thus shifting the CO flow split toward the RPA. For extracardiac TCPCs, exercise always appears to equalize the streaming, and this might be due to enhanced mixing from higher flow instabilities and turbulence at higher flow rates.

\section{A Note on SVC Flow Streaming}

Although the above results and discussion are provided in the context of preferential IVC flow streaming, they are also applicable to SVC flow streaming (based on the symmetry of the problem). An explicit relationship between SVC flow streaming and IVC flow streaming can be derived by using the principle of conservation of mass. Let $\alpha$ denote IVC flow split to the LPA, $\beta$ denote SVC flow split to the LPA, $\gamma$ denote the ratio of IVC flow to SVC flow, and $\delta$ denote CO flow split to the LPA. Then the conservation of mass principle (net mass inflow $=$ net mass outflow) requires the following equation to hold: $\beta=\delta-\gamma(\alpha-\delta)$.

In other words, the SVC flow split will be lower than the CO flow split by an amount proportional to how much IVC flow split is greater than $\mathrm{CO}$ flow split. The proportionality is equal to $\gamma$, the ratio between the IVC flow and SVC flow. If IVC and SVC flows are equal, then the equation simplifies to $\beta=2 \delta-\alpha$.

\section{CONCLUSIONS AND CLINICAL SIGNIFICANCE}

In conclusion, although the idealized TCPC study showed that both offset and $\mathrm{CO}$ split play a significant role in hepatic venous streaming, the relative contributions of these inde- pendent factors were different between intracardiac and extracardiac patient-specific TCPCs. Specifically, hepatic venous streaming in extracardiac TCPCs primarily depends on the IVC offset; $\mathrm{CO}$ split primarily governs the hepatic venous streaming in intracardiac TCPCs. More mixing between the SVC and IVC streams in intracardiac TCPCs could be the cause for the contrasting hepatic venous streaming characteristics between intracardiac and extracardiac TCPCs. Clinically, these results translate to optimizing 2 parameters, namely pulmonary artery diameters and the IVC offset in the case of extracardiac TCPC contraction. Ensuring that the pulmonary artery sizes are nearly equal without stenosis can ensure a well-balanced $\mathrm{CO}$ flow split. This is beneficial from both an energy loss standpoint and hepatic venous flow streaming. The optimization of IVC offset for extracardiac construction is a tradeoff situation. Although increasing the IVC offset can have benefits from an energy loss standpoint, it might be nonbeneficial from a hepatic venous streaming standpoint. Although the above guidelines might not directly influence the conversion from the second stage to the Fontan connection, they might help in cases related to reconstruction or repair of a Fontan connection in patients with complications secondary to poor hepatic venous effluent distribution.

\section{References}

1. Srivastava D, Preminger T, Lock JE, Mandell V, Keane JF, Mayer JE, et al. Hepatic venous-blood and the development of pulmonary arteriovenousmalformations in congenital heart-disease. Circulation. 1995;92:1217-22.

2. Duncan BW, Desai S. Pulmonary arteriovenous malformations after cavopulmonary anastomosis. Ann Thorac Surg. 2003;76:1759-66.

3. Pandurangi UM, Shah MJ, Murali R, Cherian KM. Rapid onset of pulmonary arteriovenous malformations after cavopulmonary anastomosis. Ann Thorac Surg. 1999;68:237-9.

4. Pike NA, Vricella LA, Feinstein JA, Black MD, Reitz BA. Regression of severe pulmonary arteriovenous malformations after Fontan revision and "hepatic factor'" rerouting. Ann Thorac Surg. 2004;78:697-9.

5. Shinohara T, Yokoyama T. Pulmonary arteriovenous malformation in patients with total cavopulmonary shunt: what role does lack of hepatic venous blood flow to the lungs play? Pediatr Cardiol. 2001;22:343-6.

6. Kim SJ, Bae EJ, Lee JY, Lim HG, Lee C, Lee CH. Inclusion of hepatic venous drainage in patients with pulmonary arteriovenous fistulas. Ann Thorac Surg. 2009;87:548-54 
7. Sundareswaran KS, de Zelicourt D, Sharma S, Kanter KR, Spray TL, Rossignac J, et al. Correction of pulmonary arteriovenous malformation using image-based surgical planning. JACC Cardiovasc Imaging. 2009;2:1024-30.

8. Alexi-Meskishvili V, Ovroutski S, Ewert P, Dahnert I, Berger F, Lange PE, et al. Optimal conduit size for extracardiac Fontan operation. Eur J Cardiothorac Surg. 2000;18:690-5.

9. de Zelicourt DA, Pekkan K, Wills L, Kanter K, Forbess J, Sharma S, et al. In vitro flow analysis of a patient-specific intraatrial total cavopulmonary connection. Ann Thorac Surg. 2005;79:2094-102.

10. Ensley AE, Lynch P, Chatzimavroudis GP, Lucas C, Sharma S, Yoganathan AP. Toward designing the optimal total cavopulmonary connection: an in vitro study. Ann Thorac Surg. 1999;68:1384-90.

11. Pekkan K, de Zelicourt D, Kitajima H, Forbess JM, Kanter KR, Parks JW, et al. Functional left pulmonary artery stenosis in total cavopulmonary connection (TCPC): assessing improvements in lung perfusion and cardiac workload with computer-aided angioplasty. Circulation. 2004;110:737.

12. Soerensen DD, Pekkan K, de Zelicourt D, Sharma S, Kanter K, Fogel M, et al. Introduction of a new optimized total cavopulmonary connection. Ann Thorac Surg. 2007;83:2182-90.

13. Frakes DH, Conrad CP, Healy TM, Monaco JW, Fogel M, Sharma S, et al. Application of an adaptive control grid interpolation technique to morphological vascular reconstruction. IEEE Trans Biomed Eng. 2003;50:197-206.

14. Frakes DH, Smith MJT, Parks J, Sharma S, Fogel M, Yoganathan AP. New techniques for the reconstruction of complex vascular anatomies from MRI images. $J$ Cardiovasc Magn Reson. 2005; 7:425-32.

15. KrishnankuttyRema R, Dasi LP, Pekkan K, Sundareswaran K, Fogel M, Sharma S, et al. Quantitative analysis of extracardiac versus intraatrial Fontan anatomic geometries. Ann Thorac Surg. 2008;85:810-7.

16. Salim MA, Disessa TG, Arheart KL, Alpert BS. Contribution of superior venacaval flow to total cardiac-output in children-a Doppler-echocardiographic study. Circulation. 1995;92:1860-5.
17. Whitehead KK, Pekkan K, Doddasomayajula R, Kitajima HD, Sundareswaran KS, Paridon SM, et al. Computational model of exercise effects on Fontan hemodynamics demonstrates favorable energetics in extracardiac Fontans when compared to lateral tunnel. Circulation. 2007;116:481.

18. AboulHosn J, Danon S, Levi D, Castellon Y, Child J, Moore J. Regression of pulmonary arteriovenous malformations after transcatheter reconnection of the pulmonary arteries in patients with unidirectional Fontan. Congenit Heart Dis. 2007; $2: 179-84$

19. McElhinney DB, Marshall AC, Lang P, Lock JE, Mayer JE Jr. Creation of a brachial arteriovenous fistula for treatment of pulmonary arteriovenous malformations after cavopulmonary anastomosis. Ann Thorac Surg. 2005;80: 1604-9.

20. Steinberg J, Alfieris GM, Brandt B 3rd, Smith F, Byrum CJ, Fink GW, et al. New approach to the surgical management of pulmonary arteriovenous malformations after cavopulmonary anastomosis. Ann Thorac Surg. 2003;75: 1640-2.

21. Wu IH, Nguyen KH. Redirection of hepatic drainage for treatment of pulmonary arteriovenous malformations following the Fontan procedure. Pediatr Cardiol. 2006;27:519-22.

22. Stamm C, Friehs I, Duebener LF, Zurakowski D, Mayer JE Jr, Jonas RA, et al. Improving results of the modified Fontan operation in patients with heterotaxy syndrome. Ann Thorac Surg. 2002;74:1967-77.

23. Dasi LP, Pekkan K, Katajima HD, Yoganathan AP. Functional analysis of Fontan energy dissipation. J Biomech. 2008;41:2246-52.

24. Dasi LP, Rema RK, Kitajima HD, Pekkan K, Sundareswaran KS, Fogel M, et al. Fontan hemodynamics: importance of pulmonary artery diameter. $J$ Thorac Cardiovasc Surg. 2009; 137:560-4.

25. Whitehead KK, Pekkan K, Kitajima HD, Paridon SM, Yoganathan AP, Fogel MA. Nonlinear power loss during exercise in single-ventricle patients after the Fontan-insights from computational fluid dynamics. Circulation. 2007; 116(suppl I):I165-71. 